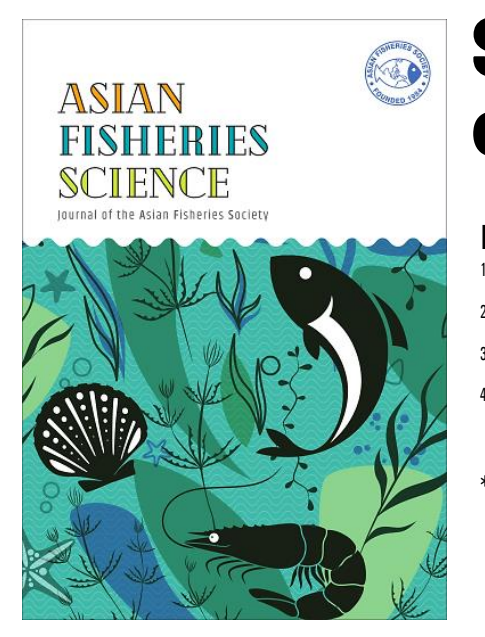

\title{
Singapore's National Action Plan on Antimicrobial Resistance
}

\author{
KELVIN LIM ${ }^{1, *}$, DIANA CHEE ${ }^{1}$, SHAWN TING ${ }^{2}$, EDMUND CHOO ${ }^{3}$, WEI LING TAN ${ }^{3}$, YUEH NUO LIN ${ }^{4}$ \\ ${ }^{1}$ Animal and Veterinary Service, National Parks Board, 52 Jurong Gateway Road, JEM Office Tower, \#09-01, Singapore 608550 \\ ${ }^{2}$ Menzies School of Health Research, Charles Darwin University, Darwin, NT 0810, Australia \\ ${ }^{3}$ Singapore Food Agency, 52 Jurong Gateway Road, JEM Office Tower, \#14-01, Singapore 608550 \\ ${ }^{4}$ Antimicrobial Resistance Coordinating Office, National Centre of Infectious Diseases, 16 Jalan Tan Tock Seng, Singapore 308422
}

*E-mail: kelvin_lim@nparks.gov.sg

(C)Asian Fisheries Society

ISSN: 0116-6514

E-ISSN: 2073-3720

https://doi.org/10.33997/j.afs.2020.33.S1.015

\section{Abstract}

The rapid emergence and spread of antimicrobial resistance (AMR) is a global threat, and international bodies have called for action against it. Studies show that Singapore is not spared from its effects. There was strong local political support for action and One Health agencies assembled a One Health AMR Workgroup to jointly develop the National Strategic Action Plan (NSAP) for Antimicrobial Resistance. The NSAP would set the framework for Singapore's response to AMR and would be aligned with the World Health Organisation's Global Action Plan on AMR with reference made to the standards and guidelines established by intergovernmental bodies such as the Food and Agriculture Organization of the United Nations and the World Organisation for Animal Health. This paper describes existing initiatives to combat AMR, and lists priority areas for further action akin to the WHO Global Action Plan for AMR. These priority areas include education, surveillance and risk assessment, research, prevention and control of infection, and optimisation of antimicrobial use. Today, the One Health AMR Workgroup comprises of the Ministry of Health, the Health Promotion Board, the National Environment Agency, National Parks Board, PUB, Singapore's National Water Agency and the Singapore Food Agency.

Keywords: antimicrobial resistance, One Health, antibiotics, aquaculture

\section{Introduction}

The rapid emergence and spread of antimicrobial resistance (AMR) is a pressing threat to health globally (Robinson et al., 2016). As microbes become increasingly resistant to antimicrobial agents, the ability to treat infections are becoming increasingly compromised. Aside from prolonged illnesses and increased mortality, AMR can lead to decreased productivity, higher treatment costs, and economic losses (O'Neill, 2018). Singapore sees similar trends in the creeping costs of AMR. In a 2012 study on the financial burden of multi-drug resistant (MDR) Gramnegative infections in Singapore hospitals, excess hospitalisation costs attributable to MDR infection was shown to be about SG\$ 8,640 per person, and $62 \%$ of the excess cost attributed to MDR infection was paid for by government subvention ( $\mathrm{Ng}$ et al., 2012). Such infections were also associated with longer hospitalisation, with the excess length of stay attributable to MDR infections of 6.1 days after adjustment for confounders (Lye et al., 2012).

AMR is a transboundary, inter-sectoral issue as it can spread across borders through international human, animal, and food movement. Resistance in each sector, e.g. human, animal, and environment, can have direct and indirect consequences on other sectors. A study in Korea showed that fishery workers exposed to antibiotic use in aquaculture had significantly higher resistance to cephalothin, tetracycline and trimethoprim-sulfamethoxazole compared to nonfishery workers (Shin and Cho, 2013). As Singapore is an international travel and transhipment hub that imports more than $90 \%$ of its food, AMR poses a threat to Singapore's public and animal health and food security. Livestock farming in Singapore is very limited and predominantly chicken layer and ruminant dairy production. In contrast, Singapore has a small but thriving and increasingly important food fish 
aquaculture industry, which currently accounts for about $10 \%$ of local food fish consumption (Lim et al., 2020), but has been rising through the years. As Singapore aims to produce $30 \%$ of the population's nutritional needs locally by 2030 (MSE, 2020), we envisage that the local aquaculture sector would continue to develop and expand in the long term.

Recognising the negative impact of AMR, Singapore joined the global call for action against AMR, beginning with the development of a multi-sectoral national plan to combat the threat of AMR. We describe here the development and implementation of Singapore's National Strategic Action Plan (NSAP) (OH AMRWG, 2017). The NSAP unifies and formalises the existing responses mounted across the animal, human, food, and environment sectors, while providing a roadmap to address existing gaps and prioritise future interventions.

\section{Approach for Developing the NSAP}

At the global level, the Food and Agriculture Organization of the United Nations (FAO), the World Health Organization (WHO), and the World Organisation for Animal Health (OIE) have agreed that addressing AMR requires a "One Health" approach (WHO, 2016). This concept of a tripartite collaboration on addressing health risks at the animal-humanecosystems interfaces was formalised in 2010. In Singapore, the One Health Coordinating Committee (OHCC) was established in 2012 and provides strategic oversight including setting priorities, reviewing One Health policies and programmes, as well as promoting inter-agency coordination and collaboration. The OHCC convened a One Health AMR Workgroup in January 2017 that composed of representatives from the Ministry of Health $(\mathrm{MOH})$, then-Agri-Food and Veterinary Authority (AVA), the National Environment Agency (NEA) and the PUB, Singapore's National Water Agency. From 1 April 2019, all food-related functions carried out by then-AVA, are carried out by a new statutory board, the Singapore Food Agency (SFA). In parallel, non-food animal and plant related functions of then-AVA are transferred to the National Parks Board (NParks). SFA is the lead agency for all matters related to food safety and security, and NParks is the lead agency for animal health, welfare, and management, as well as plant health. Both agencies continue to be part of the Workgroup. The One Health AMR Workgroup also included infectious diseases and public health professionals from public hospitals and the Saw Swee Hock School of Public Health, which were actively involved in AMR surveillance, research, and education. This One Health AMR Workgroup, led by the $\mathrm{MOH}$, compiled and coordinated efforts to combat AMR, which included the identification of priority areas for action across human, animal, food, environment, and water sectors. In addition to the Workgroup, three multi-sectoral sub-working groups were formed to help identify and coordinate cross-sectoral initiatives in education, surveillance, and research.

From the start, agencies agreed that the NSAP would set the framework for Singapore's response to AMR and would be aligned with the WHO Global Action Plan on AMR with reference made to the standards and guidelines established by intergovernmental bodies such as FAO and OIE. Following a series of meetings and discussions, the plan was launched on 01 November 2017. Today, the One Health AMR Workgroup comprises of $\mathrm{MOH}$, the Health Promotion Board (HPB, the agency which oversees public education), NEA, NParks, PUB and the SFA.

The NSAP aims to reduce the emergence and spread of drug-resistant organisms through the five core strategies: a) education, b) surveillance and risk assessment, c) research, d) prevention and control of infection, and e) optimisation of antimicrobial use.

Under each of the five strategies, the NSAP describes existing AMR initiatives by the relevant agencies while articulating gaps and priority areas for further action, which were identified as strategic areas requiring attention. Initiatives were examined to see how crosssectoral action and collective efforts with international partners and local stakeholders could be initiated, coordinated, or expanded. At the same time, the NSAP looks at specific initiatives for each key stakeholder group, which includes the public, professionals (e.g. doctors, veterinarians), and the industry (e.g. farmers, wholesalers, feed mill operators). While the overarching strategies to address AMR in the NSAP were designed with a longterm perspective, an interim time frame of five years was used for developing and implementing the initial programme and activities, with periodic review to ensure relevance and effectiveness.

\section{Implementation of the NSAP}

It was recognised in the early stages of development that a central body would be needed to coordinate the implementation of the NSAP and facilitate information sharing. In September 2018, the $\mathrm{MOH}$ established the AMR Coordinating Office (AMRCO) under the auspices of the National Centre for Infectious Diseases (NCID). Following the launch of the NSAP, a detailed multisectoral five-year work plan together with a reiterative monitoring and evaluation (M\&E) framework, was laid out by the Workgroup to coordinate and track the progress of implementation across all sectors. The NSAP M\&E framework identifies specific output and outcome indicators recommended for the monitoring of the Global Action Plan (WHO, 2015). Implementation of Singapore's NSAP is now underway; some of the initiatives under each of the five core strategies being implemented are described below. 


\section{Education}

Education is essential to ensure that all stakeholders, including the public, have a correct understanding and perception of AMR's impact on health and society, as well as what they can do to combat AMR. Surveys have shown that the public has many misconceptions about antibiotics, including the manner that they should be used (Pan et al., 2016). Agencies agreed that a national strategy should be coordinated to improve awareness and understanding of AMR and to present consistent messages to the public and target audiences. In 2018 and 2019, public education campaigns were launched to educate the public on appropriate antibiotic use, that antibiotics do not work on the flu virus and does not hasten flu recovery. One of the mainstay messages for consumers is that AMR has an impact on all stakeholders along the food chain, and therefore food safety and good hygiene during handling, cooking, and storing food is important.

Targeted education and communication campaigns were established as a priority area for further action for the professional and industry groups. Veterinarians play a critical role in promoting and ensuring the responsible use of antimicrobials for animal health and welfare. NParks maintains active engagements with the veterinary professionals through current AMR awareness events that leverage on international events such as World Veterinary Day and World Antimicrobial Awareness Week. Through seminars and AMR articles in newsletters, NParks will also work on capability building and continuing professional education development programmes to help veterinarians exercise antimicrobial stewardship. For other industry stakeholders, it was recognised that educational efforts on infection and disease control must form a big part of the efforts to promote the prudent and responsible use of antimicrobials in animals. For the food aquaculture sector, SFA maintains a voluntary farm quality assurance scheme known as the Good Aquaculture Practices for Fish Farming (GAP-FF) (SFA, 2020), which will reduce the reliance on antimicrobials through the reduction of incidence of infections. In addition, as part of SFA's efforts to raise public awareness of AMR, SFA published an informative segment on AMR on its webpage and also developed an animation video to educate the public on the importance of AMR and what they can do to reduce the spread.

\section{Surveillance and risk assessment}

Detection of AMR through surveillance, coupled with risk assessment, enables timely and appropriate response to be mounted. However, monitoring of resistance trends will require surveillance data on AMR patterns, good epidemiological information of infection rates, antimicrobial usage, and health outcomes. AMR surveillance is also necessary to measure, monitor, and evaluate the programme outcomes and overall impact of the NSAP.

A key objective of the NSAP is an integrated surveillance programme for antimicrobial resistance and utilisation across the human, animal, food, and environmental sectors. This would shed light on how specific resistance develops and spread between humans and animals, through food, water, and environment locally. As a first step towards integration, a joint report of existing surveillance data on resistance and utilisation has been published $(\mathrm{OH}$ AMRWG, 2019).

With respect to the food production and supply chain, Singapore monitors antimicrobial sales for agricultural use at the wholesalers' level, and utilisation based on antimicrobials stored at the farm. As Singapore imports most of its food, the National Centre for Food Science (NCFS), a designated OIE Collaborating Centre for Food Safety, focuses on surveillance for drug residues and monitoring of AMR bacteria in food, both locally produced and imported. Resistant pathogens monitored include multi-drug resistant (MDR) Salmonella, methicillin-resistant Staphylococcus aureus (MRSA), and extendedspectrum beta-lactamases (ESBL)-producing Escherichia coli. As one of the NSAP initiatives, existing surveillance for Salmonella enterica serovar Enteritidis in local chicken layer farms were recently extended to include all Salmonella species and E. coli in all local poultry and ruminant dairy farms. Singapore also conducts AMR testing for pathogens in diseased fish. AMR surveillance in food would subsequently be expanded to include retail food outlets, using a risk-based approach that takes into consideration antimicrobials of veterinary and human health importance in the OIE and WHO lists, respectively. The farm to fork surveillance in Singapore will better inform on the potential risk areas along the food supply chain, for targeted efforts to combat AMR.

\section{Research}

It was recognised that cross-sectoral AMR research was a gap that needed to be addressed as part of the NSAP. Consultations with the local research community had identified the following priority research areas for cross-sectoral collaborations:

a) Transmission pathways between sectors (human, animal, food, and the environment) and the implications of cross-sectoral transmission on efforts to control AMR.

b) Attitudes, practices, and knowledge of $A M R$, to determine how best to change behaviour to facilitate its control.

c) Socio-economic impact of AMR. 
A One Health AMR research grant is being established, which would specifically fund cross-sectoral research in these three focus areas.

Another priority area for further action in the aquaculture sector is to engage relevant stakeholders (local research groups, biomedical companies, feed producers, vaccine developers and academic institutions) and facilitate applied research into the development of viable alternatives to reduce the widespread use of antimicrobials in the aquaculture sector. Equally important is the collection of scientific evidence and cost-benefit studies to support and encourage the use of such alternatives. Of note is that a recently published qualitative study showed that the use of antibiotics for growth promotion was uncommon in Singapore food fish farms (Lim et al., 2020).

\section{Prevention and Control of Infection}

Every infection prevented means one less opportunity for antimicrobial use and for organisms to develop resistance. Thus, vaccination and other infection prevention and control measures are important to limit the development and spread of AMR.

The SFA and NParks currently implement both regulatory (e.g. licensing conditions, vaccine registration) and non-regulatory guidelines (e.g. good animal husbandry practices) for farms. In the future, local guidelines will be harmonised with the ASEAN (Association of Southeast Asian Nations) guidelines for Good Animal Husbandry Practices (GAHP) and Good Aquaculture Practices (GAP), developed by the ASEAN Sectoral Working Groups for Livestock and Fisheries, respectively. Local guidelines are actively promoted to the local farmers to improve animal health management practices. At the same time, Singapore will continue to highlight the benefits of vaccination and facilitate access to safe and effective vaccines of the farming industry. Several autogenous fish vaccines are currently registered and permitted for use in Singapore, and national guidelines on their use and production are being developed. In tandem, NParks is in the process of reviewing vaccine evaluation and pharmacovigilance processes.

\section{Optimisation of Antimicrobial Use}

The inappropriate (overuse and misuse) use of antimicrobials across animal and human sectors has been the biggest driver of the development of AMR in microorganisms. The NSAP explains the rationale for the recommendations for the proper regulation of health products and medicines, that is, to ensure that the right antimicrobials are used at the right time, in the right dose, and for the right duration.

The NSAP describes the various regulations, initiatives, and position statements on the regulation and optimisation of the use of antimicrobials. This includes Singapore's position on the non-therapeutic use of antimicrobials, drug residues, and licensing conditions for farms and feed mills which ban specified antimicrobials. The NSAP highlights Singapore's commitment to establish a robust regulatory framework for the supply chain control of antimicrobials and to establish a roadmap to progress towards veterinary prescriptions for drugs used in all animal sectors and antimicrobial stewardship programmes. In this aspect, current regulations are in the process of being reviewed and strengthened to support these goals.

\section{International Collaboration}

Singapore's NSAP is not just focused on local initiatives, but it also identifies potential areas of collaboration with international partners. The inclusion of this strategy in the NSAP signals Singapore's commitment to global and regional efforts to minimise the emergence and spread of AMR, because AMR is a global risk that is beyond the capacity of any organisation or nation to manage or mitigate alone. This strategy is carried out in three ways:

a) International benchmarking: Singapore is committed to participating in global surveillance networks such as the WHO global AMR surveillance system (GLASS) and OIE global database on the use of antimicrobial agents in animals. These networks and databases are important in establishing an international understanding of AMR and antimicrobial utilisation.

b) International partnerships: Aside from participating in the implementation of the Global Action Plan, Singapore is a member of the ASEAN and supports member states to improve health systems by sharing technical expertise and experience. Singapore has committed to fighting AMR at the United Nations General Assembly in New York in September 2016 as part of the collective political declaration. Singapore, together with other ASEAN member states, also declared in November 2017, its commitment to combat AMR through a multi-sectoral and multidisciplinary approach within the framework of One Health, which is consistent with Singapore's NSAP. In addition, ASEAN has endorsed Singapore's initiative to coordinate and spearhead efforts to combat AMR in the livestock and aquaculture sectors in ASEAN at the ASEAN Ministerial Meetings on Agriculture and Forestry in 2016 (AMAF, 2016) and 2017 (AMAF, 2017). At the same time, Singapore is partnering with FAO to develop guidelines on monitoring guidelines of AMR in bacterial pathogens in aquaculture, 
together with other international partners (FAO, 2018).

c) International research collaborations: It is recognised that tapping on partnerships with international health research funding agencies will enable more efficient use of research funding and resources. Recognising that any investment in new medicines, vaccines and diagnostic tools to combat AMR would be for the long-term, Singapore will continue to foster industry partnerships and facilitate industry research and development.

\section{Conclusion}

Singapore's NSAP, which incorporates strategies for the aquaculture sector, was developed by parties in four different sectors: human, animal, food, and environment, and launched within a relatively short time. Some factors that enabled this was the strong political support received from the start, and strong foundation of One Health cooperation and collaboration. Agencies were familiar with one another, had a common purpose, and agreed early on the direction to take. Self-assessment and transparency between agencies were critical to facilitate this process. Decisive leadership and the commitment of team members also contributed to its successful development. The inclusion of infectious disease and public health experts in the AMR field provided current knowledge on antimicrobial utilisation and prescribing practices in human health, as public health was a key consideration in the NSAP objectives. Work is now underway to implement the initiatives outlined in the NSAP through a five-year multi-sectoral work plan, with crucial monitoring indices identified for evaluation. The NSAP expresses Singapore's commitment to the local and international community in recognising that $A M R$ is a global problem and local efforts are part of a global solution. The NSAP would serve Singapore and the aquaculture sector well for the next five years until the next review to ensure the objectives and goals can be met.

\section{References}

AMAF, $38^{\text {th }}$ Meeting of the ASEAN Ministers on Agriculture and Forestry. 2016. https://asean.org/storage/2016/10/Annex-29-JPS-38thAMAF-final.pdf (Accessed 010 ctober 2020).

AMAF, 39 th Meeting of the ASEAN Ministers on Agriculture and Forestry. 2017. https://asean.org/storage/2017/09/8.-Joint-Press-Statement39th-AMAF-Final.pdf (Accessed 01 October 2020).

FAO (Food and Agriculture Organisation). 2018. Regional Consultation and Related Study on Antimicrobial Resistance (AMR) Risk to Aquaculture in Asia and Preliminary Consultation on Monitoring of AMR in Bacterial Pathogens in Aquaculture. 2018. http://www.fao.org/asiapacific/events/detail-events/en/c/1500/ (Accessed 01 October 2020).

Lim, J.M., Duong, M.C., Hsu, L.Y., Tam, C.C. 2020. Determinants influencing antibiotic use in Singapore's small-scale aquaculture sectors: A qualitative study. Plos one 15:e0228701.
Lye, D.C., Earnest, A., Ling, M.L., Lee, T.-E., Yong, H.-C., Fisher, D.A. Krishnan, P., Hsu, L.-Y. 2012. The impact of multi-drug resistance in healthcare-associated and nosocomial Gram-negative bacteraemia on mortality and length of stay: cohort study. Clinical Microbiology and Infection 18:502-508.

MSE (Ministry of Sustainability and the Environment). 2020. https://www.mse.gov.sg/news/mewr-cos-2020--building-aclimate-resilient-singapore-for-singaporeans--with-singaporeans-1 (Accessed 01 October 2020).

Ng, E., Earnest, A., Lye, D.C., Ling, M.L., Ding, Y., Hsu, L.Y. 2012. The excess financial burden of multi-drug resistance in severe gramnegative infections in Singaporean hospitals. Academy of Medicine, Singapore 41:189-193.

O'Neill, J. 2018. Tackling drug-resistant infections globally: Final report and recommendations. 2016. HM Government and Welcome Trust: UK. https://www.biomerieuxconnection.com/wp-content/uploads /2018/04/Tackling-Drug-Resistant-Infections-Globally_-FinalReport-and-Recommendations.pdf (Accessed 01 October 2020).

OH AMRWG (One Health Antimicrobial Resistance Workgroup). 2017. National Strategic Action Plan on Antimicrobial Resistance. https://www.ncid.sg/About-NCID/OurDepartments/AntimicrobialResistance-Coordinating-Office/Documents/National\%20Strategic \%20Action\%20Plan\%20on\%20Antimicrobial\%20Resistance.pdf (Accessed 01 October 2020).

OH AMRWG (One Health Antimicrobial Resistance Workgroup). 2019. One Health Report on Antimicrobial Utilisation and Resistance. https://www.ncid.sg/About-NCID/OurDepartments/AntimicrobialResistance-Coordinating-Office/Documents/One\%20Health \%20Report\%20on\%20Antimicrobial\%20Utilisation\%20and\%20Resi stance \%202017.pdf (Accessed 010 ctober 2020).

Pan, D.S.T., Huang, J.H., Lee, M.H.M., Yu, Y., Mark, I., Chen, C., Goh, E.H., Jiang, L., Chong, J.W.C., Leo, Y.S. 2016. Knowledge, attitudes and practices towards antibiotic use in upper respiratory tract infections among patients seeking primary health care in Singapore. BMC Family Practice 17:148.

Robinson, T.P., Bu, D.P., Carrique-Mas, J., Fèvre, E.M., Gilbert, M., Grace, D., Hay, S.l., Jiwakanon, J., Kakkar, M., Kariuki, S., Laxminarayan, R., Lubroth, J., Magnusson, U., Thi Ngoc, P., Van Boeckel, T.P., Woolhouse, M.E.J. 2016. Antibiotic resistance is the quintessential One Health issue. Transactions of the Royal Society of Tropical Medicine and Hygiene 110:377-380. https://doi.org/10.1093/trstmh/trw048

Shin, H.-H., Cho, S.-H. 2013. Prevalence of antimicrobial resistance in Escherichia coli strains isolated from fishery workers. Osong Public Health and Research Perspectives 4:72-75.

Singapore Food Agency (SFA). 2020. https://www.sfa.gov.sg/foodfarming/farming-initiatives/good-aquaculture-practices-for-fishfarming-(gap-ff) (Accessed 010 ctober 2020).

WHO (World Health Organization). 2015. Global Action Plan on Antimicrobial Resistance. https://apps.who.int/iris/bitstream /handle/10665/193736/9789241509763_eng.pdf?sequence=1 (Accessed 01 October 2020).

WHO (World Health Organization). 2016. Antimicrobial resistance: a manual for developing national action plans. https://apps.who.int /iris/handle/10665/204470 (Accessed 01 October 2020). 